\title{
Stigmasterol blocks cartilage degradation in rabbit model of osteoarthritis
}

\author{
Wei-Ping Chen, Chong Yu, Peng-Fei Hu Jia-Peng Bao, Jing-Li Tang and Li-Dong $\mathrm{Wu}^{\bowtie}$ \\ Department of Orthopedics Surgery, the 2nd Affiliated Hospital of Medical College, Zhejiang University, Hangzhou, People's Republic of China
}

\begin{abstract}
Stigmasterol has been shown exihbit anti-osteoarthritic properties in vitro studies. However, the in vivo effects of stigmasterol on cartilage are still unclear. This study investigated the anti-osteoarthritic properties of stigmasterol on cartilage degradation in a rabbit model of osteoarthritis (OA). Twenty rabbits underwent bilateral anterior cruciate ligament transection (ACLT) to induce OA. Five rabbits were used as normal control. Two weeks after operation, the rabbits were randomly divided into two groups. Each group of 10 rabbits received intra-articular injection with $0.3 \mathrm{ml}$ of stigmasterol in left knees and vehicle in right knees, once weekly. Group 1 was killed 6 weeks after ACLT and 2 were sacrificed 9 weeks after ACLT. The knee joints were assessed by gross morphology, histology and gene expression analysis. We found that expression of genes encoding matrix metalloproteinases (MMPs) was significantly higher while tissue inhibitors of metalloproteinase (TIMP)-1 was significantly lower in the both joints of the two OA groups compared to normal contrals. Stigmasterol reduced the cartilage degradation as assessed by histological analysis and markedly suppressed MMPs expression both in group 1 and group 2. Our results suggest that stigmasterol may be considered as a possible therapeutical agent in the treatment of $O A$.
\end{abstract}

Key words: matrix metalloproteinases, osteoarthritis, stigmasterol

Received: 11 August, 2011; revised: 04 June, 2012; accepted:

20 August, 2012; available on-line: 16 October, 2012

\section{INTRODUCTION}

Osteoarthritis (OA) is a chronic joint disease manifested by cartilage degradation along with severe joint pain. Chondrocytes exposed to abnormal stimuli such as mechanical damage or proinflammatory stimuli including interleukin-1 (IL-1) and tumor necrosis factor $\alpha$ $(\mathrm{TNF}-\alpha)$, release matrix metalloproteinases (MMPs), the main matrix-degrading enzymes, leading to the breakdown of extracellular matrix (ECM) (Rowan et al., 2008). MMP-13 is well known as the most effective proteinase because of the ability to cleave collagen II which is the main component of ECM (Billinghurst et al., 1997).

Treatment of $\mathrm{OA}$ is still a problem. Although nonsteroidal anti-inflammatory drugs and selective cyclooxygenase-2 (COX-2) inhibitors are effective, they have severe side effects such as gastrointestinal bleeding and cardiovascular diseases (Farkouh et al., 2004; Lazzaroni et al., 2004). There is still a need to investigate new antiarthritic agents.

Phytosterols are plant lipids whose chemical structure is similar to that of cholesterol. Previous studies have shown that phytosterols exert various biological effects such as anti-cancer, anti-pyretic and immune-modulating activities (Gupta et al., 1980; Raicht et al., 1980; Bouic et al., 1996; Bouic et al., 1999). In addition, their antiinflammatory activities are also well demonstrated (Navarro et al., 2001; Okoye et al., 2010; Youssef et al., 2010). The role of phytosterols in rheumatoid arthritis (RA) has been investigated previously (Thiers, 1953). Recently, Gabay et al demonstrated that stigmasterol, a phytosterol, exerted anti-osteoarthritic properties in mouse chondrocytes and human chondrocytes (Gabay et al., 2010). That was the first study to report a possible role of phytosterols in preventing OA. However, the in vivo effects of stigmasterol on cartilage are still unclear. Therefore, in the present study, we investigated the in vivo effects of stigmasterol on cartilage in experimental OA.

\section{MATERIAL AND METHODS}

Reagents. Stigmasterol was purchased from SigmaAldrich (St.Louis, MO, USA). Stigmasterol stock solution at a concentration of $20 \mathrm{mg} / \mathrm{ml}$ was prepared in ethanol. Then, the solution was diluted with $50 \%$ polyethylene glycol (PEG).

OA induction and animal treatment. With the approval by the Institutional Animal Care and Use Committee of Zhejiang University (Hangzhou, China), twenty-five New Zealand White rabbits (male, 2.0-2.5 kg) were used in this study. Twenty rabbits underwent bilateral anterior cruciate ligament transection (ACLT) as described previously (Yudoh et al., 2007). Five rabbit were used as normal control. Two weeks after operation, the rabbits were randomly divided into two groups. Both groups of 10 rabbits were intra-articularly injected with $0.3 \mathrm{ml}$ of stigmasterol solution $(20 \mu \mathrm{g} / \mathrm{ml}$ in PEG) in left knees and vehicle in right knees, once weekly. Group 1 was killed 6 weeks after operation and 2 were sacrificed 9 weeks after operation. The knee joints were assessed by gross morphology, histology and gene expression analysis.

Macroscopic evaluation. Scoring of the articular cartilage of the rabbit knee joints was performed in a blind manner after using India ink. The criteria used were as follows: grade 1 (intact surface), grade 2 (minimal fibrillation), grade 3 (overt fibrillation), grade 4 (erosion) (Yoshioka et al., 1997).

e-mail: Idwu@yahoo.com

Abbreviations: ACLT, anterior cruciate ligament transection; COX2, cyclooxygenase-2; DMOAD, disease modifying OA drug; ECM, extracellular matrix; EDTA, ethylenediaminetetraacetate; IL-1, nterleukin-1; MMPs, matrix metalloproteinases; OA, osteoarthritis; PEG, polyethylene glycol; RA, rheumatoid arthritis; TIMP, tissue inhibitors of metalloproteinase; TNF-a, tumor necrosis factor $a$. 
Table 1. Criteria for histologic evaluation

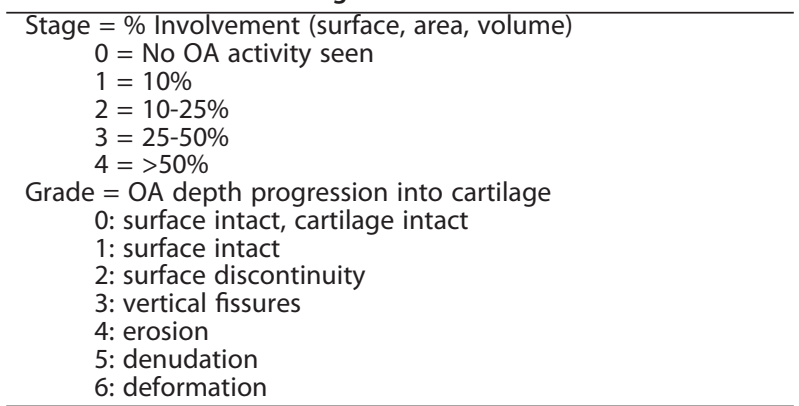

Histological assessment. Femoral condyles from both knees were fixed in 10\% neutral buffered formalin, decalcified with buffered ethylenediaminetetraacetate (EDTA) and dehydrated in a series of ethanol solutions. Then, the cartilage was embedded in paraffin, cut into $5-\mu \mathrm{m}$ sections. The sections were stained with Safranin O-fast green. The OARSI assessment system with a combined score of grade $(0-6) \times$ stage $(0-4)$ was used to evaluate the sections in a blind manner (Pritzker et al., 2006).

Gene expression analysis of cartilage. Cartilage was pulverized in liquid nitrogen, and total RNA was extracted using TRIzol reagent (Invitrogen). For the first strand cDNA synthesis, $1 \mu \mathrm{g}$ of RNA was reverse transcribed using Moloney murine leukemia virus reverse transcriptase cDNA synthesis kit (Promega, USA) at $37^{\circ} \mathrm{C}$ for $1 \mathrm{~h}$. Gene expression of MMP-1, MMP3, MMP-13 and tissue inhibitors of metalloproteinase (TIMP-1) was quantitated by real-time quantitative PCR with the iCycler apparatus system (Bio-Rad, USA). The primers used were shown in Table 2. 18S rRNA was used as an internal control. Levels of target gene expression were calculated as follows: $n=100 \times 2^{-}(\Delta C T$ targeted gene $-\Delta$ CT $18 \mathrm{~S}$ rRNA).

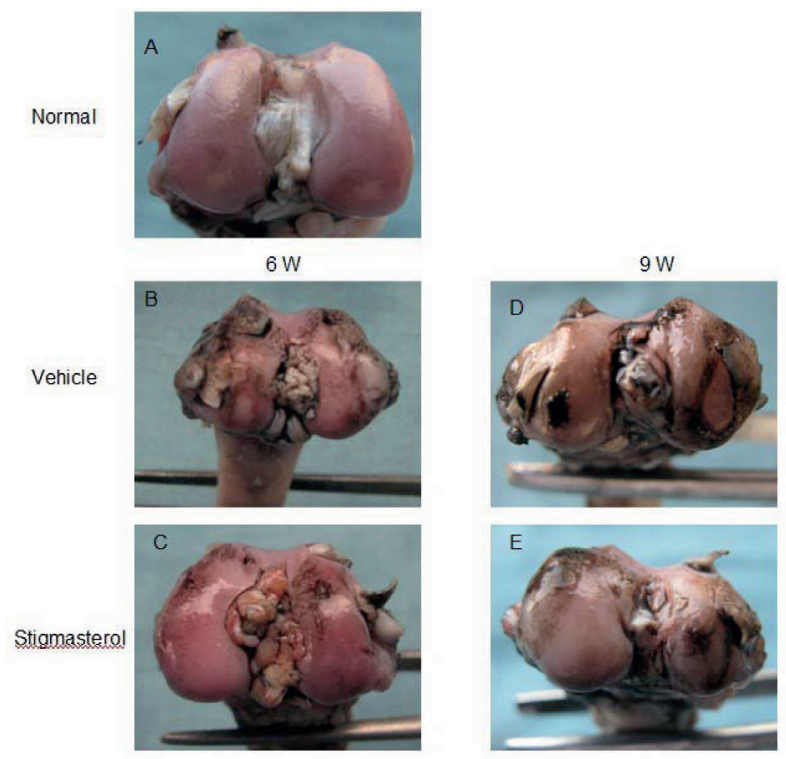

Figure 1. Gross appearance of femoral condyles

Gross morphology of femoral condyles from normal (A) and ACLT rabbits 6 and 9 weeks after operation. Femoral condyles in vehicle-treated group showed more serious lesions during the development of OA, while the lesions in stigmasterol-treated cartilage was less severe 6 weeks (B compare with C) and 9 weeks (D compare with E) after ACLT. OA = osteoarthritis; $A C L T=$ anterior cruciate ligament transection.
Statistical analysis. All data were expressed as mean \pm standard deviation (S.D.). Statistical analyses were performed with, SPSS 12.0 software for Windows. Statistical analysis of the gross morphology was performed by nonparametric Mann-Whitney $U$ test, histological and gene expression data was analyzed by a paired $t$-test. $P$ values less than 0.05 were considered significant.

\section{RESULTS}

\section{Macroscopic grading of cartilage}

The articular cartilage showed various severities of lesions. Cartilage from OA animal treated with vehicle showed significant severe lesions compared to normal cartilage. Those lesions were more severe in cartilage 9 weeks after ACLT than 6 weeks after ACLT. The stigmasterol-treated animal showed less severe cartilage lesions compared with the vehicle-treated group, both in 6 and 9 weeks after ACLT, however, the difference was not significant $(P>0.05$, data not shown). Figure 1 presents the cartilage lesions in different groups.

\section{Histological evaluation}

Representative histological pictures of control, vehicletreated $\mathrm{OA}$ and stigmasterol-treated $\mathrm{OA}$ cartilage are presented in Fig. 2, and the histological scores are shown in Table 3. In vehicle-treated knees, ACLT led to cartilage degradation. Surface irregularity and depletion were noted in the cartilage 6 weeks after ACLT. Severe degradation such as cartilage erosion was observed 9 weeks after ACLT. The cartilage degradation was significantly diminished by stigmasterol.

\section{Gene expression in cartilage}

There was a significant increase in the expression of MMP-1, MMP-3 and MMP-13 genes in the cartilage from vehicle-treated $\mathrm{OA}$ animal cartilage harvested 6 or 9 weeks after ACLT compared to normal cartilage. In contrast, expression of the TIMP-1 gene was decreased. In addition, the MMPs expression increased in parallel with the progression of OA. Furthermore, stigmasterol

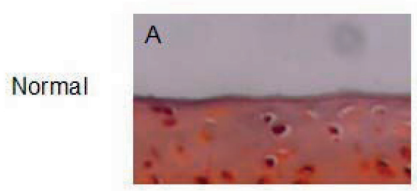

$6 \mathrm{~W}$

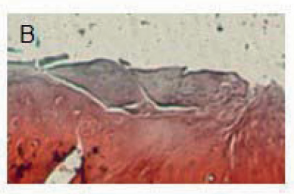

C

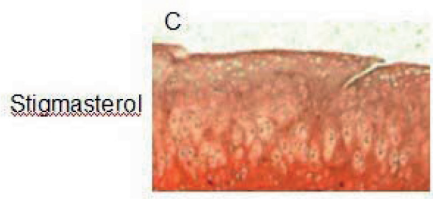

9W
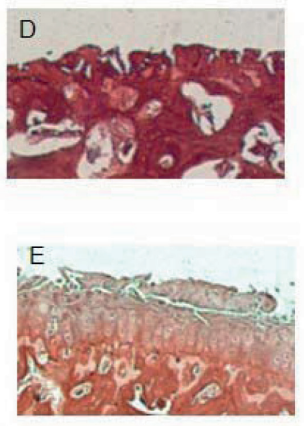

Figure 2. Safranin-O staining of articular cartilage

Safranin $\mathrm{O}$ staining was performed in sections of cartilage. Typical cartilage lesions are shown (Original magnification $\times 50$ ). 
Table 2. Primers for targeted genes

\begin{tabular}{llll}
\hline $\begin{array}{l}\text { Targeted } \\
\text { genes }\end{array}$ & Sequence $\left(5^{\prime}-3^{\prime}\right)^{*}$ & $\begin{array}{l}\text { Amplicon } \\
\text { length (bp) }\end{array}$ & $\begin{array}{l}\text { Accession } \\
\text { number }\end{array}$ \\
\hline MMP-1 & $\begin{array}{l}\text { S: GACGTGGACCGACAACAGTGA } \\
\text { A: GGGGAACATTAGTGCTCCTACATC }\end{array}$ & 112 & AH005676 \\
MMP-3 & $\begin{array}{l}\text { S: ACACCGGATCTGCCAAGAGA } \\
\text { A: CTGGAGAACGTGAGTGGAGTCA }\end{array}$ & 89 & NM001082280 \\
MMP-13 & $\begin{array}{l}\text { S: TTGACCACTCCAAGGACCCAG } \\
\text { A: GAGGATGCA GACGCCAGAAGA }\end{array}$ & 252 & NM001082037 \\
TIMP-1 & $\begin{array}{l}\text { S: CAACTGCGGAACGGGCTCTTG } \\
\text { A: CGGCAGCGTAGGTCTTGGTGAA }\end{array}$ & 102 & AY8297305 \\
$\begin{array}{l}\text { 18S } \\
\text { rRNA }\end{array}$ & $\begin{array}{l}\text { S: GACGGACCAGAGCGAAAGC } \\
\text { A: CGCAGCGGCATCGTTATG }\end{array}$ & 119 & EU236696 \\
\hline
\end{tabular}

${ }^{*} \mathrm{~S}=$ Sense; $\mathrm{A}=$ Antisense

significantly decreased the expression of the MMP-1, MMP-3 and MMP-13 genes, and increased the expression of TIMP-1 compared to the vehicle-treated group (Fig. 3).

\section{DISCUSSION}

Current treatment of $\mathrm{OA}$ is unsatisfactory and although symptom relief, no disease modifying OA drug (DMOAD) is yet available. There is still a need to find new agents for the treatment of OA.

Recently, Gabay et al. (2010) demonstrated that stigmasterol, a phytosterol, showed anti-osteoarthritic properties in both mouse chondrocytes and human chondrocytes in response to IL-1 $\beta$. Little is known about the in vivo effects of stigmasterol on OA. Therefore, we design this study to investigate whether stigmasterol exerted in vivo effects on cartilage degradation in experimental OA. In the experimental rabbit OA model induced by ACLT, treatment with stigmasterol reduced the progression of cartilage damage as assessed by morphological non-sig-
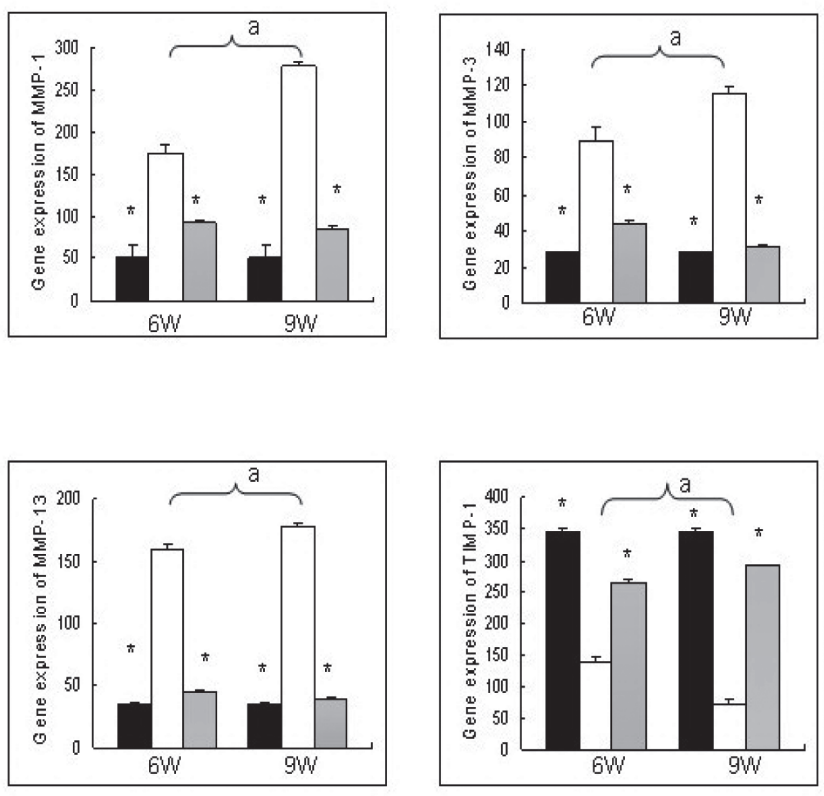

Figure 3. Gene expression of MMP-1, MMP-3, MMP-13 and TIMP-1 in cartilage Quantitative real-time PCR was used to analyze the expression of MMP-1, MMP-3, MMP-13 and TIMP-1 genes in cartilage. Data are presented as mean \pm S.D. aP<0.05. ${ }^{*} P<0.05$ compared to the vehicle-treated group. normal

$\square$ Vehicle

口Stigmasterol

nificantly and histological evaluation. In addition, treatment with stigmasterol resulted in the down-regulation of expression of MMP-1, MMP-3, MMP-13 and up-regulation of TIMP-1. These findings confirmed the in vitro effects of stigmasterol reported by Gabay and coworkers.

It is well established that numerous proteinases such as MMPs, aggrecanases and cathepsins are implicated in the catabolism of OA cartilage (Dean et al., 1989; Konttinen et al., 2002; Tortorella et al., 2001). Among them, MMPs are believed to play pivotal roles in cartilage matrix degradation. MMP-13 has received extensive attention because it is mainly responsible for type II collagen degradation. An elevated MMP-13 expression was noted in arthritic cartilage (Aigner et al., 2001). In addition, a selective MMP-13 inhibitor has been shown to reduce cartilage degradation in experimental OA induced by ACLT in rabbits and in the rat model of mono-iodoacetate (MIA)-induced OA (Johnson et al., 2007; Baragi et al., 2009). In the present study, we focused on changes of gene expression of MMPs in cartilage due to their importance in OA. The present results revealed that the expression of MMP-1, MMP-3 and MMP-13 was significantly elevated by ALCT compared to normal cartilage and this up-regulation was related to the cartilage degradation in the progression of OA. Our results are partly consistent with the findings reported by Wu and coworkers (Wu et al., 2008), who demonstrated that the level of MMP-1 expression was correlated with the degree of cartilage degradation in ACLT rabbits. In addition, we found that the expression of TIMP-1 was decreased along with the cartilage degradation. Thus, the balance between MMPs and TIMPs was disturbed. Since the imbalance between MMPs and TIMPs in the present study increased with the progressive cartilage degradation as assessed by morphology and histology, our results confirmed the important role of MMPs/TIMPs in the process of OA.

In the present study, treatment with stigmasterol by intra-articular injection resulted in the down-regulation of the expression of MMP-1, MMP-3 and MMP-13 and up-regulation of TIMP1 as compared to the vehicle-treated groups. These observations suggest that stigmasterol administration regulated the balance between MMPs and TIMPs during the progression of OA. This could be partly supported by the finding that stigmasterol inhibited the gene expression of MMP-3 and MMP-13 in mouse chondrocytes and human chondrocytes in vitro (Gabay et al., 2010). An inhibition of MMPs by phytosterol was also observed in another system as reported by Grether-Beck et al. (2008). In addition, a preparation rich in phytosterol, named avocado-soybean unsaponifiables (ASU), also has been shown to inhibit MMPs expression in experimental OA in dogs (Boileau et al., 2009). These observations suggest that phytosterol may exert inhibitory effects on MMPs in different systems. Additionally, many 
Table 3. Histological assessment of articular cartilage using OARSI assessment system

$$
\text { Vehicle Stigmasterol }
$$

\begin{tabular}{lll}
\hline 6 weeks after ACLT & $5.8 \pm 2.05$ & $2.8 \pm 1.1^{*}$ \\
9 weeks after ACLT & $13.6 \pm 2.19$ & $9.6 \pm 2.5^{*}$ \\
\hline
\end{tabular}

Data are presented as mean \pm S.D. ${ }^{*} P<0.05$.

agents showing inhibitory effects on MMPs have shown beneficial effects in experimental OA. Thus, we speculate that the beneficial effects of stigmasterol on cartilage may be partly associated with the regulation of the MMPs/TIMPs system.

The main limitations of our study are its duration (6 or 9 weeks after ACLT) and the single dosage in the study. Longer time could provide more information about the changes of gene expression of MMPs and TIMPs as well as the in vivo effects of stigmasterol in experimental OA. For instance, in earlier studies, three stage of $\mathrm{OA}$ was used to investigate the gene expression of catabolic mediators in ACLT rabbits (Mehraban et al., 1997; Bao et al., 2009). In addition, we only used one dosage in the experiment, maybe different dosage could better illustrate the effects of sigmasterol. Another limitation is that the mechanism by which stigmasterol regulated the MMPs/TIMPs in cartilage in vivo remains unclear. In vitro, stigmasterol has been shown to inhibit pro-inflammatory cytokines and matrix degradation mediators partly via inhibiting the nuclear factor- $x \mathrm{~B}$ (NF-xB) pathway (Gabay et al., 2010). The effects of stigmasterol on another important signalling pathway, mitogen-activated protein kinase (MAPK) pathway which is implicated in MMPs regulation remains unknown (Yan \& Boyd, 2007). In addition, cartilage degradation is partially dependent on MMPs activities, there are mechanisms involved in cartilage degradation indepent on MMPs, and the effects of sigmasterol on thes mechanisms are also unclear. Therefore, further studies are needed to get a better understanding about the mechanisms of action of stigmasterol in $\mathrm{OA}$.

In summary, we first reported that stigmasterol effectively inhibits cartilage degradation in a rabbit ACLT model. Our results suggest that stigmasterol may be considered as a possible therapeutical agent in the treatment of OA. Further studies are needed to elucidate the action mechanism of the regulation of MMPs by stigmasterol.

\section{Acknowledgments}

This study was supported by the National Natural Science Foundation of China (81071492).

\section{Competing interests}

The author(s) declare that they have no competing interests.

\section{REFERENCES}

Aigner T, Zien A, Gehrsitz A, Gebhard PM, McKenna L (2001) Anabolic and catabolic gene expression pattern analysis in normal versus osteoarthritic cartilage using complementary DNA-array technology. Arthritis Rheum 44: 2777-2789.

Bao JP, Chen WP, Feng J, Zhao J, Shi ZL, Huang K, Wu LD (2009) Variation patterns of two degradation enzyme systems in articular cartilage in different stages of osteoarthritis: regulation by dehydroepiandrosterone. Clin Chim Acta 408: 1-7.

Baragi VM, Becher G, Bendele AM, Biesinger R, Bluhm H, Boer J, , Deng H, Dodd R, Essers M, Feuerstein T, Gallagher BM Jr, Gege C, Hochgürtel M, Hofmann M, Jaworski A, Jin L, Kiely A, Korniski B, Kroth H, Nix D, Nolte B, Piecha D, Powers TS, Richter
F, Schneider M, Steeneck C, Sucholeiki I, Taveras A, Timmermann A, Van Veldhuizen J, Weik J, Wu X, Xia B (2009) A new class of potent matrix metalloproteinase 13 inhibitors for potential treatment of osteoarthritis: Evidence of histologic and clinical efficacy without musculoskeletal toxicity in rat models. Arthritis Rheum 60: 2008-2018.

Billinghurst RC, Dahlberg L, Ionescu M, Reiner A, Bourne R, Rorabeck C, Mitchell P, Hambor J, Diekmann O, Tschesche H, Chen J, Van Wart H, Poole AR (1997) Enhanced cleavage of type II collagen by collagenases in osteoarthritic articular cartilage. J Clin Invest 9: $1534-1545$.

Boileau C, Martel-Pelletier J, Caron J, Msika P, Guillou GB, Baudouin C, Pelletier JP (2009) Protective effects of total fraction of avocado/soybean unsaponifiables on the structural changes in experimental dog osteoarthritis: inhibition of nitric oxide synthase and matrix metalloproteinase-13. Arthritis Res Ther 11: R41.

Bouic PJ, Etsebeth S, Liebenberg RW, Albrecht CF, Pegel K, Van Jaarsveld PP (1996) beta-Sitosterol and beta-sitosterol glucoside stimulate human peripheral blood lymphocyte proliferation: implications for their use as an immunomodulatory vitamin combination. Int J Immunopharmacol 18: 693-700.

Bouic PJ, Lamprecht JH: Plant sterols and sterolins (1999) a review of their immune-modulating properties. Altern Med Rev 4:170-177.

Dean DD, Martel-Pelletier J, Pelletier JP, Howell DS, Woessner JF, Jr (19890 Evidence for metalloproteinase and metalloproteinase inhibitor imbalance in human osteoarthritic cartilage. I Clin Invest 84: 678-685.

Farkouh ME, Kirshner H, Harrington RA, Ruland S, Verheugt FW, Schnitzer TJ, Burmester GR, Mysler E, Hochberg MC, Doherty M, Ehrsam E, Gitton X, Krammer G, Mellein B, Gimona A, Matchaba P, Hawkey CJ, Chesebro JH (2004) TARGET Study group: comparison of lumiracoxib with naprox en and ibuprofen in the therapeutic arthritis research and gastrointestinal event trial (TARGET), cardiovascular outcomes: randomised controlled trial. Lancet 364: 675-684.

Gabay O, Sanchez C, Salvat C, Chevy F, Breton M, Nourissat G, Wolf C, Jacques C, Berenbaum F(2010) Stigmasterol: a phytosterol with potential anti-osteoarthritic properties. Osteoarthritis Cartilage 18: 106-116.

Grether-Beck S, Muhlberg K, Brenden H, Krutmann J (2008) Topical application of vitamins, phytosterols and ceramides. Protection against increased expression of interstital collagenase and reduced collagen-I expression after single exposure to UVA irradiation. Hautar:t 59: 557-562.

Gupta MB, Nath R, Srivastava N, Shanker K, Kishor K, Bhargava KP (1980) Anti-inflammatory and antipyretic activities of beta-sitosterol. Planta Med 39: 157-63.

Johnson AR, Pavlovsky AG, Ortwine DF, Prior F, Man CF, Bornemeier DA, Banotai CA, Mueller WT, McConnell P, Yan C, Baragi V, Lesch C, Roark WH, Wilson M, Datta K, Guzman R, Han HK, Dyer RD (2007) Discovery and characterization of a novel inhibitor of matrix metalloprotease-13 that reduces cartilage damage in vivo without joint fibroplasia side effects. J Biol Chem 282: 27781-2791.

Konttinen YT, Mandelin J, Li TF, Salo J, Lassus J, Liljestrom M, Hukkanen M, Takagi M, Virtanen I, Santavirta S (2002) Acidic cysteine endoproteinase cathepsin $\mathrm{K}$ in the degeneration of the superficial articular hyaline cartilage in osteoarthritis. Arthritis Rheum 46: 953960.

Lazzaroni M, Bianchi Porro G (2004) Gastrointestinal side-effects of traditional non-steroidal anti-inflammatory drugs and new formulations. Aliment Pharmacol Ther 20 (Suppl 2): 48-58.

Mehraban F, Tindal MH, Proffitt MM, Moskowitz RW (1997) Temporal pattern of cysteine endopeptidase (cathepsin B) expression in cartilage and synovium from rabbit knees with experimental osteoarthritis: gene expression in chondrocytes in response to interleukin-1 and matrix depletion. Ann Rheum Dis 56: 108-115.

Navarro A, De las Heras B, Villar A (2001) Anti-inflammatory and immunomodulating properties of a sterol fraction from Sideritis foetens Clem. Biol Pharm Bull 24: 470-473.

Okoye FB, Osadebe PO, Proksch P, Edrada-Ebel RA, Nworu CS, Esimone CO (2010) Anti-inflammatory and membrane-stabilizing stigmastane steroids from Alchornea floribunda leaves. Planta Med 76: $172-177$.

Pritzker KP, Gay S, Jimenez SA, Ostergaard K, Pelletier JP, Revell PA, Salter D, van den Berg WB (2006) Osteoarthritis cartilage histopathology: grading and staging. Osteoarthritis Cartilage 14: 13-29.

Raicht RF, Cohen BI, Fazzini EP, Sarwal AN, Takahashi M (1980) Protective effect of plant sterols against chemically induced colon tumors in rats. Cancer Res 40: 403-405.

Rowan AD, Litherland GJ, Hui W, Milner JM (2008)Metalloproteases as potential therapeutic targets in arthritis treatment. Expert Opin Ther Targets 12: 1-18.

Thiers H (1953) The value of stigmasterol (R. Wulzen's antistiffness factor essential for guinea pigs) in the therapy of rheumatic diseases. Rev Rhum Mal Osteoartic 20: 636-643. 
Tortorella MD, Malfait AM, Deccico C, Arner E (2001) The role of ADAM-TS4 (aggrecanase-1) and ADAM-TS5 (aggrecanase-2) in a model of cartilage degradation. Osteoarthritis Cartilage 9: 539-552.

Wu H, Du J, Zheng Q (2008) Expression of MMP-1 in cartilage and synovium of experimentally induced rabbit ACLT traumatic osteoarthritis: immunohistochemical study. Rheumatol Int 29: 31-36.

Yan C, Boyd DD (2007) Regulation of matrix metalloproteinase gene expression. I Cell Physiol 211: 19-26.

Yoshioka M, Shimizu C, Harwood FL, Coutts RD, Amiel D (1997) The effects of hyaluronan during the development of osteoarthritis. Osteoarthritis Cartilage 5: 251-260.
Youssef DT, Ibrahim AK, Khalifa SI, Mesbah MK, Mayer AM, van Soest RW (2010) New anti-inflammatory sterols from the Red Sea sponges Scalarispongia aqabaensis and Callyspongia siphonella. Nat Prod Commun 5: 27-31.

Yudoh K, Shishido K, Murayama H, Yano M, Matsubayashi K, Takada H, Masuko K, Kato T, Nishioka K (2007) Water-soluble C60 fullerene prevents degeneration of articular cartilage in osteoarthritis via down-regulation of chondrocyte catabolic activity and inhibition of cartilage degeneration during disease development. Arthritis Rheum 56: 3307-3318. 